Pacific Journal of Mathematic

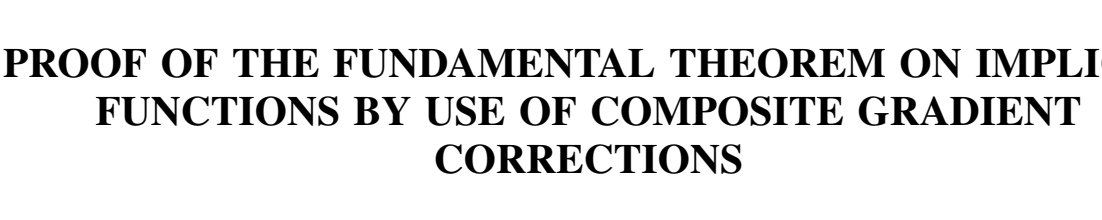




\title{
PROOF OF THE FUNDAMENTAL THEOREM ON \\ IMPLICIT FUNCTIONS BY USE OF COMPOSITE GRADIENT CORRECTIONS
}

\author{
William L. Hart and Theodore S. Motzkin
}

1. Introduction. Many methods have been employed for establishing the classical result, Theorem 2.1, concerning the existence of functions $x_{i}(t)$ satisfying a system

$$
f_{j}(x ; t)=0, \quad(j=1,2, \cdots, n)
$$

of $n$ equations in $n$ unknowns $\left(x_{1}, \cdots, x_{n}\right)=x$ with $\left(t_{1}, \cdots, t_{p}\right)=t$, where all variables and functions are real valued, and $f_{j}(\alpha ; \beta)=0$. The object of this article is to present a new proof of the theorem by a constructive method of successive approximations involving corrections related to the gradients in $x$-space of the functions $f_{j}(x ; \beta)$.

To establish Theorem 2.1, a sequence $x^{(m)}(t)$ with $x^{(0)}(t)=\alpha$ will be defined, where $x^{(m)}(t)$ is obtained by adding to $x^{(m-1)}(t)$ a vector correction $\Delta x^{(m-1)}(t)$ which is equal to a certain constant, $\rho$, times the vector sum of corrections parallel to the gradients of the $f_{j}(x ; \beta)$ at $x=\alpha$. The vector $\Delta x^{(m-1)}(t)$, for a fixed $t$, is a special case of the corresponding correction of an iterative process for solving a general system $g_{j}(x)=0$, $(j=1, \cdots, k), k \geqq n$, introduced by the authors in a previous article [2].

For a particular system (1.1), the method of the present paper would be applicable to obtaining values of the $x_{i}(t)$ by use of a digital computing machine for any $t$ sufficiently near $t=\beta$. Section 6 in [2] describes a related small arc method with the same objective; the two methods differ in the values of the arguments used in fundamental matrices which appear with similar roles in [2] and below. The method of [2] might be superior computationally to the method of the present paper. However, in $\S 6$ in [2], Theorem 2.1 below was employed as a starting point. Thus the present paper shows that the composite gradient method is effective to establish the supporting Theorem 2.1 as well as the related small arc method of [2] for computing values of the implicit functions.

In connection with the present article, it is pertinent to mention the proof of Theorem 2.1 by E. Goursat, [1], extended by William L. Hart, [3] and [4], to various infinite systems. In the Goursat method for (1.1), a system

Received March 27, 1958. Presented to the American Mathematical Society, April 19, 1958. Sponsored in part by the Office of Naval Research; reproduction permitted for all purposes of the U.S. Government. 


$$
x_{j}=\Phi_{j}(x ; t)
$$$$
(j=1,2, \cdots, n)
$$

equivalent to (1.1) is constructed by use of the inverse of the matrix ${ }^{1}$ $A=\left(a_{i j}\right), a_{i j}=\partial f_{j}(\alpha ; \beta) / \partial x_{i}$; then a solution $x(t)$ of $(1.2)$ is defined by applying the method of successive substitutions to (1.2). In contrast, under the same hypotheses as those of Goursat, $\S 2$ employs a system (1.2) constructed by direct use of $A$, without forming its inverse. This feature might be of computational advantage. In case $n=1$, the present method with $\rho=1$ is identical with the Goursat method.

Either Goursat's method or the present method can also be regarded as a constructive algorithm solving the problem of elimination of $n-1$ variables $x_{1}, \cdots, x_{n-1}$ from $n$ equations $f_{j}(x ; t)=0$ leading to a relation (such as $x_{n}=x_{n}(t)$ ) between the remaining variables $\left(x_{n}, t_{1}, \cdots, t_{p}\right)$.

The problem of solving $y=F(x ; u), F=\left(F_{1}, \cdots, F_{n}\right)$ and $y=\left(y_{1}\right.$, $\left.\cdots, y_{n}\right)$, by $x=\phi(y ; u)$ (inversion with and without parameters), for nonzero Jacobian $F_{x}$, is only apparently more general than the solution of (1.1) (to subsume it set $t=(y, u), f=F-y)$ and thus is equally amenable to our iterative procedure.

2. Construction of a system (1.2) equivalent to (1.1). We shall consider (1.1) subject to the following hypotheses:

$$
\begin{aligned}
& \text { The } f_{j} \text { are continuous, and all derivatives } \partial f_{j} / \partial x_{i} \text { exist and } \\
& \text { are continuous in some open neighborhood } \Omega \text { of }(x=\alpha ; t=\beta) . \\
& \qquad f_{j}(\alpha ; \beta)=0 \quad(j=1,2, \cdots, n) .
\end{aligned}
$$

$$
f_{j}(\alpha ; \beta)=0
$$

(2.3) The matrix $A=\left(a_{i j}\right), a_{i j}=\partial f_{j}(\alpha ; \beta) / \partial x_{i}$, is nonsingular.

In $x$-space, let the positive gradient of $f_{j}(x ; \beta)$ at $x=\alpha$ be defined as having the magnitude $\left(\sum_{i=1}^{n} a_{i j}^{2}\right)^{1 / 2}$, nonzero because of (2.3), and the direction angles $\Psi_{i j}$ specified by

$$
\cos \Psi_{i j}=a_{i \jmath} w_{\jmath}^{-1} ; \quad w_{\jmath}=\left(\sum_{i=1}^{n} a_{i j}^{2}\right)^{\frac{1}{2}} .
$$

For any $(x ; t)$ and each $j$ define, formally, a vector correction $\Delta_{j} x$ for $x$, where $x$ is considered an approximation to a solution of $f_{j}(x ; t)=0$, by specifying the $i$ th component $\Delta_{j} x_{i}$ of $\Delta_{j} x$ as follows:

$$
\Delta_{j} x_{i}=-\rho f_{j}(x ; t) w_{j}^{-1} \cos \Psi_{i j},
$$

with a constant $\rho>0$ to be restricted later. Then define the composite vector correction $\Delta x$ for $x$, considered now as an approximation to a solution of (1.1), by specifying for $\Delta x$ the $i$ th component

1 Capital italic letters represent $n$ by $n$ matrices. The transpose of a matrix $A$ is denoted by $A^{\prime}$. We treat $x$ as a one-rowed matrix. 


$$
\Delta x_{i}=\sum_{j=1}^{n} \Delta_{j} x_{i} ; \text { or, } \Delta x=\sum_{j=1}^{n} \Delta_{j} x .
$$

By use of (2.6) we introduce, formally, a sequence $x^{(m)}(t)$ of approximations to a solution of (1.1):

$$
x^{(0)}(t)=\alpha ; x^{(m)}(t)=x^{(m-1)}(t)+\Delta x^{(m-1)}(t), m>0 .
$$

From (2.5), the $i$ th coordinate $x_{i}^{(m)}(t)$ is given by

$$
x_{i}^{(m)}(t)=x_{i}^{(m-1)}(t)-\rho \sum_{j=1}^{n} a_{i j} w_{j}^{-2} f_{j}\left(x^{(m-1)}(t) ; t\right) .
$$

Let the components $\Phi_{i}$ of a vector $\Phi=\left(\Phi_{1}, \Phi_{2}, \cdots, \Phi_{n}\right)$ be defined by

$$
\Phi_{i}(x ; t)=x_{i}-\rho \sum_{j=1}^{n} a_{i j} w_{j}^{-2} f_{j}(x ; t) .
$$

Then (2.7) is the sequence of approximations $x^{(m)}(t)$ arising if the method of successive substitutions, with $x^{(0)}(t)=\alpha$, is used to seek a solution of the system

$$
x=\Phi(x, t) .
$$

By use of (2.1) and (2.3) we find that (1.1) and (2.10) are equivalent systems.

We remark that, in $x$-space, $\Delta x$ of (2.6) is invariant under an orthogonal transformation of coordinates and under an alteration of $f_{j}(x ; t)$ to $h_{j} f_{j}(x ; t)$ if $h_{j} \neq 0$. Thus, before considering the existence and convergence of sequence (2.7), we may assume that (1.1) has been altered by dividing $f_{j}(x ; t)$ by $w_{j}$ of $(2.4)$. Then, without change of notation, from (2.4) and (2.5) for all $j$ we obtain

$$
w_{j}=1 ; \Delta_{j} x_{i}=-\rho a_{i j} f_{j}(x ; t) ; \sum_{i=1}^{n} a_{i j}^{2}=1 .
$$

Note that $A A^{\prime}$ is symmetric and positive definite Hence there exist positive characteristic constants $\lambda_{1}, \cdots, \lambda_{n}$ and an orthogonal matrix $S=\left(s_{p q}\right)$ such that

$$
S A A^{\prime} S^{\prime}=\left(\delta_{i j} \lambda_{i}\right)=D \text {, where } \delta_{i i}=1 \text { and } \delta_{i j}=0 \text { if } i \neq j .
$$

Now, in (1.1), let the coordinates be changed from $\left(x_{1}, \cdots, x_{n}\right)$ to $\left(z_{1}, \cdots, z_{n}\right)=z$ by the orthogonal transformation $x=z S$. Then, with $g_{j}(z ; t)=f_{j}(x ; t)$ when $x=z S$, and $\alpha=\gamma S$ or $\gamma=\alpha S^{\prime}$, (1.1) becomes

$$
g_{j}(z ; t)=0, \text { where } g_{j}(\gamma ; \beta)=0, \quad(j=1,2, \cdots, n) .
$$

If we let $b_{i j}=\partial g_{j}(\gamma ; \beta) / \partial z_{i}$ and $B=\left(b_{i j}\right)$, we have

$$
B=S A ; \quad B B^{\prime}=S A A^{\prime} S^{\prime}=D ; \quad B^{\prime} B=A^{\prime} A .
$$


From $B^{\prime} B=A^{\prime} A$ on comparing main diagonal terms we obtain $\sum_{i=1}^{n} b_{i j}^{2}=$ $\sum_{i=1}^{n} a_{i j}^{2}=1$, for all $j$. Hence, if sequence (2.7) is formed for (2.13) by use of the analogue of (2.11) in the $z$-coordinates, from (2.8) we arrive at

$$
\left\{\begin{array}{c}
z^{(0)}(t)=\gamma ; z^{(m)}(t)=z^{(m-1)}(t)+\Delta^{(m-1)}(t), m>0 \\
\Delta z_{i}^{(m-1)}(t)=-\rho \sum_{j=1}^{n} b_{i j} g_{j}\left(z^{(m-1)}(t) ; t\right)
\end{array}\right.
$$

On account of the invariant features which were mentioned concerning the gradient corrections $\Delta x^{(m-1)}(t)$ of (2.7) for (1.1), it follows that the existence of all $z^{(m)}(t)$ for any $t$ is equivalent to the existence of all $x^{(m)}(t)$ and that $x^{(m)}(t)$ and $z^{(m)}(t)$ represent the same point. We shall find it convenient to discuss $z^{(m)}(t)$ instead of $x^{(m)}(t)$.

We introduce the functions

$$
\phi_{h}(z ; t)=z_{h}-\rho \sum_{j=1}^{n} b_{h j} g_{j}(z ; t) \quad(h=1, \cdots, n),
$$

and consider the following system, obtained as in (2.10), which is equivalent to $(2.13)$ :

$$
z_{h}=\phi_{h}(z ; t)
$$$$
(h=1, \cdots, n) .
$$

In (2.17) the $\phi_{k}$ and all derivatives $\partial \phi_{h} / \partial z_{i}$ are continuous when $(z ; t)$ is in $\Omega$, now defined with coordinates $(z ; t)$. With $\phi=\left(\phi_{1}, \cdots, \phi_{n}\right)$, sequence (2.15) can be written

$$
z^{(0)}(t)=\gamma ; \quad z^{(m)}(t)=\phi\left(z^{(m-1)}(t) ; t\right) .
$$

From (2.16) and $B B^{\prime}=D$ we obtain $\frac{\partial \phi_{h}}{\partial z_{i}}=\delta_{h i}-\rho \sum_{j=1}^{n} b_{h j} \frac{\partial g_{j}}{\partial z_{i}}$;

$$
\begin{gathered}
\frac{\partial \phi_{h}(\gamma ; \beta)}{\partial z_{h}}=1-\rho \sum_{j=1}^{n} b_{h j} b_{h j}=1-\rho \lambda_{h} ; \\
\frac{\partial \phi_{h}(\gamma ; \beta)}{\partial z_{i}}=0, \quad \text { if } h \neq i .
\end{gathered}
$$

Let $\mu_{i}=1-\rho \lambda_{i}$ and $\sigma_{\rho}=\max _{i \leq n}\left|\mu_{i}\right|$. Since $\left(\lambda_{1}, \cdots, \lambda_{n}\right)$ are the characteristic constants of $A A^{\prime}$ and $\sum_{i=1}^{n} a_{i j}^{2}=1$ for all $j$, we have

$$
\sum_{i=1}^{n} \lambda_{i}=n ; \quad 0<\lambda_{i}<n \text {, if } n>1 .
$$

Then the following lemma can be proved easily as in $[2]^{2}$.

LEMMA 2.1. In order that $\sigma_{\rho}<1$, it is necessary that $0<\rho<2$, and it is sufficient that

\footnotetext{
2 See formulas (4.16)-(4.18) in [2] with $r=\omega=n$.
} 
PROOF OF THE FUNDAMENTAL THEOREM ON IMPLICIT FUNCTIONS

$$
0<\rho \leqq 2 / n
$$$$
(0<\rho<2 / n \text { if } n=1) .
$$

Moreover, the minimum value of $\sigma_{\rho}$ occurs for a single value $\rho=\rho_{0}$ where

$$
-\frac{2}{n}<\rho_{0}<\frac{2(n-1)}{n} \text { if } n>2 \text {, and } \rho_{0}=1 \text { if } n \leqq 2 \text {. }
$$

For each $t$, and $i=1,2, \cdots, n$, let $\left(z=\xi^{(i)} ; t\right)$ be a point in $\Omega$ and define

$$
v_{k i}(t)=\frac{\partial \phi_{i}\left(\xi^{(i)} ; t\right)}{\partial z_{h}}-\delta_{k i}\left(1-\rho \lambda_{i}\right)
$$

Let $V(t)=\left(v_{h i}(t)\right)$, and introduce the following matrices :

$$
\begin{gathered}
B_{\rho}=I-\rho D=\left(\delta_{h i}\left(1-\rho \lambda_{i}\right)\right) ; \\
U(t)=B_{\rho} V^{\prime}(t)+V(t) B_{\rho}+V(t) V^{\prime}(t)=\left(u_{i j}(t)\right) .
\end{gathered}
$$

Note that $u_{i j}(t)$ is a polynomial with each term of degree 1 or 2 in the elements $v_{i j}(t)$ of $V(t)$. Let $H(t)=\left[\sum_{i, j=1}^{n} u_{i j}^{2}(t)\right]^{1 / 2}$.

LEMma 2.2. Select $\rho>0$ so that $\sigma_{\rho}<1$, and choose $\theta>0$ with $\sigma_{\rho}<$ $\theta<1$. Then there exist $\varepsilon>0$ and $\delta \leqq \varepsilon, \delta>0$, such that, if ${ }^{3}\|t-\beta\| \leqq \delta$, $\|z-\gamma\| \leqq \varepsilon$, and all $\left\|\xi^{(i)}-\gamma\right\| \leqq \varepsilon$ in $(2.21)$, then the functions $g_{j}(z ; t)$, $\partial g_{j}(z ; t) / \partial z_{i}$, and $z^{(1)}(t)$ exist and are continuous, and

$$
\begin{gathered}
\left\|z^{(1)}(t)-\gamma\right\| \leqq \varepsilon(1-\theta) ; \\
0 \leqq H(t) \leqq \theta^{2}-\sigma_{\rho}^{2} .
\end{gathered}
$$

To establish Lemma 2.2 first notice that, if $t=\beta$ and all $\xi^{(i)}=\gamma$ in (2.21), then all $v_{i j}(t)=0$ and thus all $u_{i j}(t)=0$. Hence $\varepsilon>0$ exists so that the specified conditions are satisfied by the $g_{i}, \partial g_{j} / \partial z_{i}$, and $H(t)$ if $\|z-\gamma\| \leqq \varepsilon,\|t-\beta\| \leqq \varepsilon$, and all $\left\|\xi^{(i)}-\gamma\right\| \leqq \varepsilon$ in (2.21). From (2.18), $z^{(1)}(t)=\phi(\gamma ; t)$ and thus $z^{(1)}(\beta)=\gamma$. Hence, if $\delta$ is sufficiently small and $0<\delta \leqq \varepsilon$, we have (2.24) when $\|t-\beta\| \leqq \delta$. This completes the proof of Lemma 2.2.

THEOREM 2.1. Suppose that $\rho>0$ and is such that $\sigma_{\rho}<1$. Assume that (2.1), (2.2), and (2.3) are satisfied. Then there exist $\varepsilon>0$ and $\delta>0, \delta \leqq \varepsilon$, such that, if $\|t-\beta\| \leqq \delta$, all $x^{(m)}(t)$ of $(2.7)$ exist, are continuous, and satisfy $\left\|x^{(m)}(t)-\alpha\right\| \leqq \varepsilon$. Also there exists, uniformly for $\|t-\beta\| \leqq \delta$,

${ }^{3}$ For any vector $z$ we use $\|z\|$ for the length. Thus, $\|z\|=\left(\sum_{i=1}^{n} z_{i}^{2}\right)^{1 / 2}$. 


$$
\lim _{m \rightarrow \infty} x^{(m)}(t)=x(t),
$$

where $x=x(t)$ satisfies (1.1). Moreover, if a point $(x ; t)$ with $\|x-\alpha\| \leqq \varepsilon$ and $\|t-\beta\| \leqq \delta$ satisfies (1.1), then $x=x(t)$.

To establish Theorem 2.1, we shall prove the corresponding facts for the sequence $z^{(m)}(t)$ of (2.15) and system (2.17). Let $\rho, \theta$, $\varepsilon$, and $\delta$ be determined by Lemma 2.2 and, hereafter, assume that $\|t-\beta\| \leqq \delta$. Then $z^{(0)}(t)$ and $z^{(1)}(t)$ exist in the region $\|z-\gamma\| \leqq \varepsilon$; by (2.24), since $z^{(0)}(t)=\gamma$, the following inequalities are true when $k=1$ :

$$
\left\|z^{(k)}(t)-\gamma\right\| \leqq \varepsilon ;\left\|z^{(k)}(t)-z^{(k-1)}(t)\right\| \leqq \varepsilon \theta^{k-1}(1-\theta) .
$$

Assume now, for $m>1$, that $z^{(k)}(t)$ has been proved to exist, to be continuous, and to satisfy (2.26) when $k=1,2, \cdots,(m-1)$. Then $z^{(m)}(t)$ exists and is continuous; also, by the mean value theorem with respect to $\left(z_{1}, \cdots, z_{n}\right)$ for fixed $t$,

$$
\begin{aligned}
z_{i}^{(m)}(t) & -z_{i}{ }^{(m-1)}(t)=\phi_{i}\left(z^{(m-1)}(t) ; t\right)-\phi_{i}\left(z^{(m-2)}(t) ; t\right) \\
& =\sum_{h=1}^{n} \frac{\partial \phi_{i}\left(\xi^{(m, i)}(t) ; t\right)}{\partial z_{h}}\left[z_{h}{ }^{(m-1)}(t)-z_{h}{ }^{(m-2)}(t)\right],
\end{aligned}
$$

where $\xi^{(m, i)}(t)$ is a properly chosen point in $z$-space on the line segment joining $z^{(m-2)}(t)$ and $z^{(m-1)}(t)$. With $\xi^{(i)}=\xi^{(m, i)}(t)$ in (2.21), let $V(t)$ be the matrix with elements $v_{h i}(t)$, and let $U(t)$ be defined by (2.23). Note that $\left\|\xi^{(m, i)}(t)-\gamma\right\| \leqq \varepsilon$. Then, from (2.27),

$$
\left\{\begin{array}{l}
z^{(m)}(t)-z^{(m-1)}(t)=\left(z^{(m-1)}(t)-z^{(m-2)}(t)\right)\left(B_{\rho}+V(t)\right) ; \\
\left\|z^{(m)}(t)-z^{(m-1)}(t)\right\|^{2}=\left(z^{(m)}(t)-z^{(m-1)}(t)\right)\left(z^{(m)}(t)-z^{(m-1)}(t)\right)^{\prime} \\
=\left(z^{(m-1)}(t)-z^{(m-2)}(t)\right)\left(B_{\rho}^{2}+U(t)\right)\left(z^{(m-1)}(t)-z^{(m-1)}(t)\right)^{\prime} .
\end{array}\right.
$$

On applying the Cauchy inequality twice ${ }^{4}$ to the term involving $U(t)$ in (2.28), we find

$$
\begin{gathered}
\left\|z^{(m)}(t)-z^{(m-1)}(t)\right\|^{2} \leqq\left\|z^{(m-1)}(t)-z^{(m-2)}(t)\right\|^{2}\left[\sigma_{\rho}^{2}+H(t)\right] \\
\leqq \theta^{2}\left\|z^{(m-1)}(t)-z^{(m-2)}(t)\right\|^{2} .
\end{gathered}
$$

From (2.26) for $k=1,2, \cdots,(m-1)$ and (2.29), we obtain (2.26) for $k=m$. Thus, by induction, all $z^{(m)}(t)$ are defined and satisfy $(2.26)$ if $\|t-\beta\| \leqq \delta$. From (2.26), the series $\sum_{m=1}^{\infty}\left[z_{i}^{(m)}(t)-z_{i}^{(m-1)}(t)\right]$ is termwise dominated by the series $\sum_{m=1}^{\infty} \varepsilon(1-\theta) \theta^{m-1}$, and hence converges uniformly. Thus the sequence $z^{(m)}(t)$ approaches a limit, $z(t)$, uniformly for $\|t-\beta\| \leqq \delta$. Since all $z^{(m)}(t)$ are continuous, $z(t)$ is continuous. It follows from $z^{(m)}(t)=\phi\left(z^{(m-1)}(t) ; t\right)$ that $z=z(t)$ satisfies $z=\phi(z ; t)$.

4 As follows: [ $\left.\sum_{i=1}^{n} a_{i} \sum_{j=1}^{n} u_{i j} a_{j}\right]^{2} \leqq\left(\sum_{i=1} a_{i}^{2}\right) \sum_{i=1}^{n}\left(\sum_{j=1}^{n} u_{i j} a_{j}\right)^{2} \leqq\left(\sum_{i=1}^{n} a_{i}^{2}\right)^{2} \sum_{i, j=1}^{n} u_{i j}^{2}$. 
To prove that $z(t)$ is the unique solution of $(2.13)$, suppose that $(\hat{z} ; t)$ satisfies (2.13) for $\|\hat{z}-\gamma\| \leqq \varepsilon$ and $\|t-\beta\| \leqq \delta$ and assume that $\hat{z} \neq z(t)$. Then, from $\hat{z}=\phi(\hat{z} ; t)$ and $z(t)=\phi(z(t) ; t)$, by details duplicating the proof of (2.29), we have

$$
\|\hat{z}-z(t)\| \leqq \theta\|\hat{z}-z(t)\|<\|\hat{z}-z(t)\|,
$$

which contradicts the assumption that $\hat{z} \neq z(t)$. Hence the proof of Theorem 2.1 is complete, because the point $z^{(m)}(t)$ in $n$-space is the same point as $x^{(m)}(t)$, and the region $\|x-\alpha\| \leqq \varepsilon$ is the same as the region $\|z-\gamma\| \leqq \varepsilon$.

Note 2.1. With a different arrangement of details, we could arrive at Theorem 2.1 with rectangular neighborhoods $\left\{\left|t_{i}-\beta_{i}\right| \leqq \delta\right.$ for all $\left.i\right\}$ and $\left\{\left|x_{i}-\alpha_{i}\right| \leqq \varepsilon\right.$ for all $\left.i\right\}$ replacing the spherical neighborhoods $\|t-\beta\| \leqq \delta$ and $\|x-\alpha\| \leqq \varepsilon$.

Note 2.2. In use of the sequence $\left\{x^{(m)}(t)\right\}$ in any particular case to obtain approximate values of $x(t)$, flexibility is introduced through the presence of the somewhat arbitrary constant $\rho$. Greater flexibility could be introduced (as in $\S 5$ of [2]) by permitting suitably restricted variation in $\rho$, with $\rho=\rho^{(m)}$ at the $m$ th iteration; revised details would establish Theorem 2.1. with this change.

Note 2.3. Suppose that $(\alpha ; \beta)$ is not a solution of (1.1.). With only (2.1) and (2.3) as hypotheses, there exists $\varepsilon>0$ so that the region $(\|x-\alpha\| \leqq \varepsilon,\|t-\beta\| \leqq \varepsilon)$ is in $\Omega$ and (2.25) is true when $\|t-\beta\| \leqq \varepsilon$ and all $\xi^{(i)}$ of $(2.21)$ satisfy $\left\|\xi^{(i)}-\gamma\right\| \leqq \varepsilon$, as in Lemma 2.2. Now assume that

$$
\|\Phi(\alpha ; \beta)-\alpha\|<\varepsilon(1-\theta) .
$$

Then, with $x^{(0)}(t)=\alpha$, there exists $\delta \leqq \varepsilon, \delta>0$, such that, if $\|t-\beta\|$ $\leqq \delta, x^{(1)}(t)$ exists and

$$
\left\|z^{(1)}(t)-\gamma\right\|=\left\|x^{(1)}(t)-\alpha\right\|=\|\Phi(\alpha ; t)-\alpha\| \leqq \varepsilon(1-\theta),
$$

which is (2.24). Thus, with hypothesis (2.30) replacing (2.2) and $\delta$ defined as above, $\left\{x^{(m)}(t)\right\}$ converges as specified in Theorem 2.1 even when $(\alpha ; \beta)$ is not a solution of $(1.1)$

\section{REFERENCES}

1. É. Goursat, Sur la théorie des fonctions implicites, Bull. Soc. Math. France 31 (1903), 184-192. Also, cf. Gilbert A. Bliss, Princeton Colloquium Lectures on Mathematics, American Mathematical Society (1913), 16-18.

2. William L. Hart and Theodore S. Motzkin, A composite Newton-Raphson gradient method 
for the solution of systems of equations, Pacific J. Math. 6 (1956), 691-707.

3. William L. Hart, Differential equations and implicit functions in infinitely many variables, Trans. Amer. Math. Soc. 18 (1917), 125-160.

4. - . Functions of infinitely many variables in Hilbert space, Trans. Amer. Math. Soc. 22 (1922), 30-50.

UNIVERSITY OF MINNESOTA

UNIVERSITY OF CALIFORNIA, LOS ANGELES 


\section{PACIFIC JOURNAL OF MATHEMATICS}

\section{EDITORS}

David Gilbarg

Stanford University

Stanford, California

R. A. Beaumont

University of Washington

Seattle 5, Washington

\author{
A. L. Whiteman
}

University of Southern California

Los Angeles 7, California

E. G. Straus

University of California

Los Angeles 24, California

\section{ASSOCIATE EDITORS}

\author{
E. F. BECKENBACH \\ C. E. BURGESS \\ M. HALL \\ E. HEWITT
}

\author{
A. HORN \\ V. GANAPATHY IYER \\ R. D. JAMES \\ M. S. KNEBELMAN
}

L. NACHBIN
I. NIVEN
T. G. OSTROM
H. L. ROYDEN

M. M. SCHIFFER

G. SZEKERES

F. WOLF

K. YOSIDA

\section{SUPPORTING INSTITUTIONS}

\author{
UNIVERSITY OF BRITISH COLUMBIA \\ CALIFORNIA INSTITUTE OF TECHNOLOGY \\ UNIVERSITY OF CALIFORNIA \\ MONTANA STATE UNIVERSITY \\ UNIVERSITY OF NEVADA \\ OREGON STATE COLLEGE \\ UNIVERSITY OF OREGON \\ UNIVERSITY OF SOUTHERN CALIFORNIA
}

\author{
STANFORD UNIVERSITY \\ UNIVERSITY OF UTAH \\ WASHINGTON STATE COLLEGE \\ UNIVERSITY OF WASHINGTON \\ AMERICAN MATHEMATICAL SOCIETY \\ CALIFORNIA RESEARCH CORPORATION \\ HUGHES AIRCRAFT COMPANY \\ THE RAMO-WOOLDRIDGE CORPORATION
}

Mathematical papers intended for publication in the Pacific Journal of Mathematics should be typewritten (double spaced), and the author should keep a complete copy. Manuscripts may be sent to any of the editors. All other communications to the editors should be addressed to the managing editor, E. G. Straus at the University of California, Los Angeles 24, California.

50 reprints per author of each article are furnished free of charge; additional copies may be obtained at cost in multiples of 50 .

The Pacific Journal of Mathematics is published quarterly, in March, June, September, and December. The price per volume (4 numbers) is $\$ 12.00$; single issues, $\$ 3.50$. Back numbers are available. Special price to individual faculty members of supporting institutions and to individual members of the American Mathematical Society: $\$ 4.00$ per volume; single issues, $\$ 1.25$.

Subscriptions, orders for back numbers, and changes of address should be sent to Pacific Journal of Mathematics, 2120 Oxford Street, Berkeley 4, California.

Printed at Kokusai Bunken Insatsusha (International Academic Printing Co., Ltd.), No. 6, 2-chome, Fujimi-cho, Chiyoda-ku, Tokyo, Japan.

\section{PUBLISHED BY PACIFIC JOURNAL OF MATHEMATICS, A NON-PROFIT CORPORATION}

The Supporting Institutions listed above contribute to the cost of publication of this Journal, but they are not owners or publishers and have no responsibility for its content or policies. 


\section{Pacific Journal of Mathematics}

\section{Vol. 8, No. 3 \\ May, 1958}

Michael Israel Aissen, A set function defined for convex plane domaines... . 383

Robert Ellis, Distal transformation groups ................... 401

Ciprian Foias, On a commutative extension of a commutative Banach algebra ....................................... 407

Jerry William Gaddum, Linear inequalities and quadratic forms ......... 411

Allen A. Goldstein and Elliott Ward Cheney, Jr., A finite algorithm for the solution of consistent linear equations and inequalities and for the Tchebycheff approximation of inconsistent linear equations...........

William L. Hart and T. S. Motzkin, Proof of the fundamental theorem on implicit functions by use of composite gradient corrections .......... 429

Henry Berge Helson, Conjugate series and a theorem of Paley .......... 437

Wu-Chung Hsiang, Abelian groups characterized by their independent subsets....................................... 447

John W. Lamperti, On the isometries of certain function-spaces ........ 459

Karel DeLeeuw and Walter Rudin, Extreme points and extremum problems

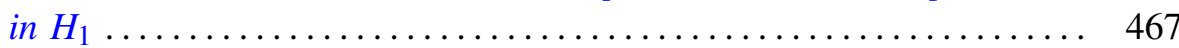

Eugene Lukacs, Some extensions of a theorem of Marcinkiewicz........ 487

George W. Mackey, Multiplicity free representations of finite groups ..... 503

Eben Matlis, Injective modules over Noetherian rings ............. 511

John William Neuberger, Continuous products and nonlinear integral equations

Lawrence Edward Payne and Hans F. Weinberger, New bounds for solutions of second order elliptic partial differential equations...

William T. Reid, A Prüfer transformation for differential systems ........ 575

Howard L. Rolf, The free lattice generated by a set of chains ...

K. M. Saksena, Inversion and representation theorems for a generalized

Laplace integral....................................... 597

Daniel Shanks, Two theorems of Gauss......................... 609

Paul Slepian, On the Lebesgue area of a doubled map ............... 613

Otto Szász and Nelson Paul Yeardley, Jr., The representation of an analytic function by general Laguerre series . ..................... 621

Alan C. Woods, On two-dimensional convex bodies ................. 635 